\title{
Precision Medicine: A New Era
}

\author{
Lisa M. Giles ${ }^{1}$. David L. Cooper ${ }^{1,2}$
}

Published online: 31 October 2018

c) Springer Nature Switzerland AG 2018

The Cancer Moonshot program [1] set the stage for elevating the importance of precision medicine (PM) as "an emerging approach for disease treatment and prevention that takes into account individual variability in genes, environment, and lifestyle for each person" [2]. Implementation of PM as a medical model has wide-reaching implications for changing standards of care and the underpinnings of how the life science industry develops therapeutics and targets patients. Continuing advances in the identification of the molecular mechanisms responsible for the pathogenesis of cancer, regardless of site of origin, have opened the potential for broad application of DNA and RNA therapeutics across cancer types. Additionally, the evolving success of new immunotherapies is an example of imprecise medicine as clinicians are trying to understand who will and will not respond. The DNA, RNA and cell-based immunotherapies used in combination are becoming the future standard of cancer treatment. In addition, the role of big data, artificial intelligence, and the integration of an array of 'omic' platforms promises the implementation of precision therapies for future patients in their fight against cancer.

\section{Precision Medicine (PM) is a Scientific and Clinical Evolution, Not a Revolution}

An indicator of this evolution is reflected in the National Cancer Institute's (NCI's) Precision Medicine Initiative (PMI) change in treatment selection protocol between their first and second trials [3]. The first NCI-Molecular Analysis for Therapy Choice (NCI-MATCH) clinical trial assigned adult patients to ten targeted treatment arms. The assignment

David L. Cooper

DLCooper12451@hotmail.com

Lisa M. Giles

lgiles@gilesaa.com

1 Giles \& Associates Consultancy, Inc., Lake Bluff, IL, USA

2 Realize Therapeutics, Chicago, IL, USA of patients to clinical trial arms based on therapeutic inhibition of driver genetic alterations advanced through MATCH Trial-1 delivered less than expected results. Yet the outcomes data were deemed an exciting step: "These findings represent a large collection of data in populations of patients who may not have been studied in conventional clinical trials, and they will have important implications for future precision medicine trials."

The NCI's PMI subsequently designed MATCH Trial-2, currently underway, with patient accrual for 24 treatment arms selected from over 4000 variants identified across 143 genes. Trial- 2 is expected to demonstrate benefits by:

- Recognizing the importance of identifing the evolution of sub-clonal cancer populations.

- Applying deep sequencing to identify rare driver mutations associated with therapeutic resistance.

- Expanding development of liquid biopsy technologies to non-invasively, serially monitor development of additional genetic alterations.

The scope of PM has broadened as we understand more about cancer pathobiology. The MATCH Trial-2 plays a vital role in demonstrating how continuing pathobiologic and technologic advances can improve patient outcomes through targeted monitoring and treatments.

The insights gained from understanding the evolution of driver genetic alterations, improves predictability and impact on patient and therapy selection. One example of PM's potential to shift the current paradigm is in the approach to single-agent cancer treatment. The cost of multiple novel oncology agents is not economically scalable, and therefore a growing need exists to improve the selection of responders and allow appropriate combination therapy earlier in treatment. Such PM advances can also help payers identify which patients will actually benefit from these expensive oncology therapies.

Researcher Dr. Pia Kvistborg, at Netherlands Cancer Institute, observes that, "In the future, cancer treatment is likely to be a combination of traditional treatments, such 
as chemotherapy, and new immunotherapies. For that reason it's important for scientists to know how immunotherapy treatment forms function at the molecular level so that we can combine treatments to achieve the most effective result" [4]. This cannot be accomplished without PM insights.

\section{PM is Impacting the Life Sciences Industry Across Discovery, Development, and Commercialization}

The effects of PM have already refreshed various life science sectors by offering the opportunity to develop new products and technologies. To drive significant improvement in cancer patient outcomes, it becomes scientifically critical to understand the tumor-specific target and how it evolves. Tumors are not static as they spread and become resistant to therapy, and this knowledge influences how cancer researchers design and conduct smarter, more focused trials. Clinicians increasingly utilize PM 'omics' capabilities to select, monitor, and reassign patients-thanks to improved discovery and development advances. For example:

1. Novel therapies, designed against multiple biomarker targets which identify specific carcinogenesis mechanisms, can determine efficaciousness earlier. With these tools, the biopharma sector can test earlier and more frequently, accelerating the assessment of target-directed therapies in development. An example is the growing activity in RNA therapeutics, including microRNA (miRNA), small interfering RNA (siRNA) and messenger RNA (mRNA) vaccines. There are over 700 DNA and RNA therapeutics in drug development pipelines, $35 \%$ of which target oncology, with RNA interference (RNAi) and RNA antisense technologies dominating the market [5]. Growth in this activity is accelerating as gene editing technologies transition from pharmaceutical development to clinical studies [6].

2. Liquid biopsy analysis will monitor the cancer to detect loss of early efficaciousness or initial refractoriness with identification of sub-clonal resistance development [7, 8]. Many liquid biopsy approaches include analysis of an increasing number of biomarker classes (i.e., exosomes, miRNA, and others) and have enabled multiple start-up companies.

3. Multi-parametric, positron emission tomography/magnetic resonance (PET-MR) molecular imaging is being advanced as part of PM as it has major advantages in the ability to generate multiple quantitative images from a single examination. Examples of benefits are the identification of:
- Patients with a high likelihood of disease recurrence following treatment.

- Metastatic disease that would render patients unsuitable for surgery.

- Low-risk sub-populations for whom surveillance can be avoided [9].

Data structure and data management will significantly advance development of software applications and analytic strategies, e.g., virtual representation of cancer biology and therapeutic intervention. PM management companies will look to grow substantially by serving private practice oncologists as well as non-academic affiliated hospital systems.

\section{Patients will Increasingly Benefit from PM Advances and Applications}

PM's original premise was to match an individual's tumor DNA to a specific therapy. PM is transitioning to benefit patients in three major areas:

1. Access to early clinical trials: Where validated effective therapy options are not available, new hope of efficacious therapy in development is possible through access to first-in-man and phase I clinical trials. PM enables clinical studies, sufficient in size for clinical trial advancement, due to establishment of patient cohorts built across multiple cooperative institutions and based on innovative drugs matched to tumor genotype/phenotype. With the advent of basket studies, patients gain broader access to the most promising treatment in clinical development.

2. Immunotherapy clinical applications: The growing field of immunotherapy shows extraordinary promise in assisting or replacing standard chemotherapy. The early success of checkpoint inhibitors has brought greater attention and investment to other areas as well, including advances beyond checkpoint inhibitors [10]:

- Activation of T-cell receptors by various approaches (e.g., bi-specific antibodies) to overcome the suppression of the cytotoxic T-cell response allowing tumor proliferation.

- Targeting of inducible T-cell co-stimulator (ICOS) to promote T-cell function and antitumor response.

- Control of the immune response against cancer by the tumor microenvironment.

3. Predictive response: Newly diagnosed patients will be treated by a combination of drugs designed for both a specific driver mutation and to generate significant and specific immune responses. Patients will further benefit 
from the increased understanding of mechanisms associated with resistance, sub-clonal proliferation and/or morbidity associated with current multiple drug combination therapy. These developments reflect a second generation in PM, beyond first-generation PM driver mutation/drug matching.

The confluence of big data and cognitive machine learning is playing a crucial role as we improve our understanding and ability to integrate data fields associated with patients, their medical histories, treatments, and outcomes. Nextgeneration PM will use combined data from multiple technologies (i.e., next-generation sequencing, proteomics [11], and metabolomics) and biomarker classes to stratify patients more appropriately $[12,13]$.

Many factions across the industry are exploring how to continually redesign and apply the fundamentals of PM to benefit patients. Over the next 10 years, the utility will not be limited to the few, but will be integral to defining value across the care spectrum, from preventive to end-of-life care. Practically, broad implementation of PM will require significant cost reduction to scale.

\section{Compliance with Ethical Standards}

Conflict of interest LG and DC have no conflicts to report.

Funding No funding to report.

\section{References}

1. Cancer Moonshot. NIH National Cancer Institute. https://www. cancer.gov/research/key-initiatives/moonshot-cancer-initiative. Accessed April 2015.

2. What is precision medicine. https://ghr.nlm.nih.gov/primer/preci sionmedicine/definition. Accessed April 2015.
3. NCI-MATCH trial (Molecular Analysis for Therapy Choice). NIH National Cancer Institute. https://www.cancer.gov/about-cancer/ treatment/clinical-trials/nci-supported/nci-match. Accessed 21 Aug 2018.

4. Sjogren K. Why Immunotherapy is such an effective cancer killer. ScienceNordic. http://sciencenordic.com/why-immunotherapysuch-effective-cancer-killer. Accessed 8 Jan 2015.

5. Gousseinov E, Kozlov M, Scanlan C, Hammons A, Bei L, Benchek Y, Pirani K, Pattnaik P, Waghmare R. Bioprocessing technology trends of RNA-based therapeutics and vaccines. BioPharm Int. 2016;29(7)30-36, 48; http://www.biopharminternationa 1.com/bioprocessing-technology-trends-rna-based-therapeuticsand-vaccines.

6. Keeler AM, ElMallah MK, Flotte TR. Gene therapy 2017: progress and future directions. Clin Transl Sci. 2017;10:242-8.

7. Daya SA, Mahfouz R. Circulating tumor DNA, liquid biopsy, and next generation sequencing: a comprehensive technical and clinical applications review. Meta Gene. 2018;17:192-201.

8. Tanos R, Thierry A. Clinical relevance of liquid biopsy for cancer screening. Transl Cancer Res. 2018;7(Suppl 2):S105-29. http:// tcr.amegroups.com/article/view/19860/pdf.

9. Miles KA, Voo SA, Groves AM. Additional clinical value for PET/MRI in oncology: moving beyond simple diagnosis. J Nucl Med. 2018. https://doi.org/10.2967/jnumed.117.203612.

10. Gong J, Chehrazi-Raffle A, Reddi S, Saglia R. Development of PD-1 and PD-L1 inhibitors as a form of cancer immunotherapy; a comprehensive review of registration trials and future considerations. J ImmunoTher Cancer. 2018;6:8. https://doi.org/10.1186/ s40425-018-0316-z.

11. Emilsson V, et al. Co-regulatory networks of human serum proteins link genetics to disease. Science. 2018;1:1. https://doi. org/10.1126/science.aaq1327.

12. Vasaikar S, Straub P, Wang J, Zhang B. Linked omics: analyzing multi-omics data within and across 32 cancer types. Nucleic Acids Res. 2018;46(Database issue):D956-63. https://www.ncbi.nlm. nih.gov/pmc/articles/PMC5753188/.

13. Dimitrakopoulos C, Hindupur SK, Hafliger L, Berh J, Montazeri H, Hall M, Beerenwinkel. Network-based integration of multi-omics data for prioritizing cancer genes. Bioinformatics. 2018;24(14):2441-48. https://academic.oup.com/bioinformatics/ article/34/14/2441/4934938. 